\title{
Erros de prescrições médicas em drogaria
}

\author{
Prescriptions errors in a drugstore
}

Recebido em: 27/11/2015

Aceito em: $11 / 03 / 2016$
Tiago Aparecido Maschio de LIMA ${ }^{1,2}$; Maria Ivonete da Silva GOUVEIA²; Luis Lenin Vicente PEREIRA²; Moacir Fernandes de GODOY ${ }^{1}$

${ }^{1}$ Faculdade de Medicina de São José do Rio Preto, Famerp, Avenida Brigadeiro Faria Lima, 5416, Vila São Pedro, CEP 15090-000, São José do Rio Preto, SP, Brasil. ${ }^{2}$ União das Faculdades dos Grandes Lagos, Unilago, Rua Doutor Nielsem, 960, Jardim Aeroporto, CEP 15030-070, São José do Rio Preto,SP, Brasil. E-mail: tiagomaschio.farmacip@gmail.com

\begin{abstract}
Exploratory, descriptive study with the aim to check the errors rate in medical prescriptions in a private drugstore. During a period of 30 days, it was collected 120 copies of medical prescriptions and were evaluated the variables legibility, prescriber identification and patient, dose and posology, abbreviations, and adequacy of legislation. In total 426 errors were identified. The most frequent errors were illegible or barely legible prescriptions (68.33\%); incomplete or missing prescriber identification $(10 \%)$; incomplete or missing patient identification $(42.5 \%)$ ); incomplete or missing dosage (40\%); incomplete or missing dose per dosage unit (45\%). Also were observed the use of prohibited abbreviations $(84.17 \%)$, and $65 \%$ of the orders did not agree with the Brazilian legislation. It was noted a high error rate in the analyzed orders, showing the need for the evaluation the Pharmacist to detect these errors and to request corrections and to prevent the risk of patient exposure to the problems caused by these errors such as adverse reactions, drug interactions, poisoning, worsening of disease or death. Furthermore, it requires the awareness to compliance of good prescribing practices, and it is suggested to carry out studies to develop strategies that minimize the occurrence of prescription errors.
\end{abstract}

Keywords: drug prescriptions; inappropriate prescribing; medication errors; pharmacy

\section{RESUMO}

Estudo descritivo exploratório com objetivo de verificar a taxa de erros em prescrições médicas coletadas em uma drogaria privada. Durante 30 dias foram coletadas 120 prescrições e analisadas as variáveis legibilidade, identificação do prescritor e paciente, dose e posologia, abreviaturas, e adequação à legislação. No total, foram identificados 426 erros. Os erros mais frequentes foram prescrições ilegíveis ou pouco legíveis $68,33 \%$, identificação do prescritor incompleta ou omissa $10 \%$, identificação do paciente incompleta ou omissa $42,5 \%$, posologia incompleta ou omissa $40 \%$, dose incompleta ou omissa $45 \%$, presença de abreviaturas $84,17 \%$, e $65 \%$ das prescrições não cumpriram de forma adequada com a legislação. Foi observada elevada taxa de erros nas prescrições analisadas, demonstrando a necessidade da avaliação do farmacêutico para a detecção destes erros, solicitação de correções e prevenção do risco de exposição do paciente aos problemas ocasionados por esses erros tais como reações adversas, interações medicamentosas, intoxicações, agravamento de doenças ou óbito. Ademais, é necessária a conscientização para o cumprimento das boas práticas de prescrição e sugere-se a realização de estudos para elaborar estratégias que minimizem a ocorrência de erros de prescrição.

Palavras-chave: prescrições de medicamentos; prescrição inadequada; erros de medicação; farmácia. 


\section{INTRODUÇÃO}

A prescrição medicamentosa é um documento elaborado por um profissional legalmente habilitado, destinada ao paciente, e sujeita à análise do farmacêutico. Deve conter informações farmacoterapêuticas sobre o medicamento a ser dispensado. Possui valor legal, pelo qual se responsabilizam os profissionais que prescrevem, dispensam e administram os medicamentos $(1,2)$.

Trata-se de um instrumento essencial para a promoção do Uso Racional de Medicamentos, compreendendo a prescrição apropriada de acordo com a legislação vigente, a farmacoeconomia, a dispensação correta, o consumo nas doses indicadas em intervalos definidos e no período de tempo adequado. Além da eficácia, segurança e qualidade que devem ser comprovadas para os medicamentos, a prescrição é considerada um importante fator para o uso racional destes. Entretanto, o ato da prescrição está sujeito às influências do prescritor, das expectativas do paciente e da indústria farmacêutica (3).

Outro fator que influencia na avaliação da qualidade da prescrição é o processo de cuidados assistenciais aos pacientes, principalmente, a Atenção Farmacêutica durante a dispensação de medicamentos. Além disso, o grande arsenal de fármacos e produtos comerciais disponíveis no mercado, os frequentes lançamentos e a intensa taxa de interações medicamentosas e de reações adversas faz com que esta importante etapa do processo de atendimento seja susceptível a erros (4).

Os erros de medicação são eventos frequentes e acarretam consequências clinicamente significantes, e, sobretudo, elevam os custos para o sistema de saúde. Os erros de prescrição são os mais sérios dentre aqueles relacionados ao ciclo da Assistência Farmacêutica. Erro de prescrição com significado clínico é definido como erro de decisão ou de redação, não intencional, que pode reduzir a probabilidade do tratamento ser efetivo ou aumentar o risco de danos ou lesão ao paciente, quando comparado com as práticas clínicas baseadas em evidências científicas e de acordo com a legislação vigente (5).

O farmacêutico é o responsável pela avaliação das prescrições e somente deve ser aviada a receita que apresentar as informações exigidas pela legislação vigente tais como: nome e endereço residencial do paciente, nome do medicamento, forma farmacêutica, posologia, dose, via de administração, duração do tratamento, data, assinatura e carimbo do profissional, endereço do consultório e o número de inscrição no respectivo Conselho Profissional, ausência de rasuras e emendas, prescrição a tinta em português, em letra de forma, clara e legível ou impressa (6).

A atual legislação brasileira estabelece algumas normas para as prescrições com o objetivo de garantir a sua qualidade, sendo necessário possuir boa legibilidade, cla- reza e inteligibilidade, ausência de rasuras e abreviaturas. No entanto, constantemente são evidenciadas falhas em relação ao preenchimento das prescrições ocasionando trocas de medicamentos, administração incorreta, problemas de adesão, e elevação dos custos do tratamento. A prescrição está sujeita a diversos aspectos que resultam em diferentes desfechos. Esse processo se origina no momento do diagnóstico médico e prescrição da farmacoterapia, finalizando-se após a dispensação do medicamento e o início do tratamento farmacológico pelo paciente, sendo essas, etapas predispostas a erros que afetam o tratamento proposto (7).

Contudo, a prescrição é o principal instrumento de comunicação entre o prescritor, o farmacêutico e o paciente. Para ser considerada apropriada, além do cumprimento da legislação, deve garantir a racionalidade, a segurança e o custo-efetividade, contribuindo para desfechos positivos no tratamento de pacientes. Os erros oriundos das prescrições médicas acarretam graves danos à saúde dos pacientes, sendo, portanto, indispensável a implantação de estratégias de identificação e prevenção. Em geral, os profissionais envolvidos com o problema não estão capacitados para identificar erros (8).

Esses erros passam despercebidos e, em consequência disso, não são registrados, sendo mal avaliados e estudados. Ademais, são considerados uma problemática relacionada à baixa competência, vergonha ou punição. Tais fatores dificultam a realização de estudos, notificação de erros, discussão das causas e implantação de soluções que minimizem sua ocorrência. Portanto, estudos são necessários para a discussão do assunto e implantação de medidas de prevenção de erros (9). Nesse contexto, este trabalho teve como objetivo verificar a taxa de erros em prescrições médicas coletadas em uma drogaria privada.

\section{MATERIAL E MÉTODOS}

Trata-se de um estudo descritivo, exploratório com abordagem quantitativa e qualitativa, através da análise de erros em 120 prescrições médicas retidas em uma drogaria de rede privada localizada na região central da cidade de São José do Rio Preto, no Estado de São Paulo, Brasil. A coleta das prescrições ocorreu no período entre 20 de maio de 2015 e 20 de junho do mesmo ano.

Cópias das prescrições foram coletadas e enumeradas. A avaliação baseou-se na metodologia dos trabalhos dos pesquisadores Rosa e cols. (2009) e Lucas e cols. (2012), por meio da verificação de sete variáveis nas prescrições: legibilidade das prescrições, identificação do prescritor, identificação do paciente, dose do medicamento, posologia do medicamento, presença de abreviaturas, e cumprimento da legislação vigente $(1,10)$ (Quadro 1). 
Quadro 1. Variáveis e critérios utilizados para avaliação de prescrições médicas $(1,10)$ de uma drogaria localizada em São José do Rio Preto, São Paulo, Brasil.

\begin{tabular}{l|l} 
Variável & Critérios de Avaliação
\end{tabular}

Grafia com boa legibilidade: lida normalmente sem problemas para entendimento da escrita; Grafia ilegivel: impossível entendimento da escrita dos medicamentos prescritos ou $50 \%$ das

Legibilidade das prescrições* demais palavras, números, símbolos e abreviaturas;

Grafia pouco legivel: mais tempo para ser lida, e não havia certeza da compreensão total de todas as palavras, números, símbolos e abreviaturas.

Completa: receituários assinados ou rubricados, com carimbo, nome do prescritor, número do Conselho Regional de Medicina (CRM), endereço do consultório ou residência e número de

Identificação do prescritor

Identificação do paciente

Dose do Medicamento**

Posologia do medicamento**

\section{Abreviaturas}

\section{Adequação a Portaria 344/98}

telefone legiveis;

Incompleta: todas as outras identificações do prescritor que não atenderam as especificações da prescrição completa;

Omissa: ausência de identificação do prescritor.

Completa: contém o nome completo e legível do paciente, juntamente com seu endereço;

Incompleta: omissão ou incompreensão dos sobrenomes e na ausência do endereço;

Omissa: identificação ausente ou ilegível.

Omissa: doses ilegíveis na totalidade dos medicamentos prescritos ou ausência das mesmas;

Incompleta: ilegibilidade ou ausência da dose de pelo menos um medicamento, em receituários com mais de um medicamento prescrito;

Completa: presença das doses completas e com legibilidade.

Omissa: posologias ausentes ou ilegíveis em todos os medicamentos prescritos;

Incompleta: ilegibilidade ou ausência da posologia de pelo menos um medicamento em receituários com mais de um medicamento prescrito;

Completa: presença da posologia completa e legível em todos os medicamentos prescritos.

Presente: ocorrência de abreviações da forma farmacêutica, via de administração, quantidade e intervalo entre doses;

Ausente: ausência das abreviações acima citadas.

Adequadas: concordância com a portaria;

Inadequadas: desacordo com a portaria.

* cada palavra foi examinada e avaliada separadamente, procurando evitar a interpretação ou dedução, sendo estabelecido, portanto, que a prescrição deve ser entendida e não interpretada; ** foram observados a nomenclatura e o sistema de pesos e medidas oficiais.

As prescrições foram coletadas por uma estudante, e posteriormente foi realizada uma dupla checagem por dois Farmacêuticos docentes, todos do curso de Farmácia. O contato com o prescritor para adequação das receitas, quando necessário, foi realizado de acordo com a rotina do estabelecimento.

Os dados das prescrições médicas foram transferidos para um banco de dados utilizando a planilha do software Excel (2010). Foi realizada uma análise estatística descritiva, utilizando o próprio Excel, visando caracterizar as variáveis encontradas nas prescrições. As variáveis categóricas foram apresentadas com números e proporções (\%).

O presente estudo foi aprovado pelo Comitê de Ética em Pesquisa (CEP) da União da Faculdade dos Grandes Lagos (Unilago), sob o parecer número 118/15 atendendo aos aspectos preconizados pela Resolução número 466/2012 do Conselho Nacional de Saúde no que se refere ao sigilo dos dados e à divulgação dos resultados apenas para fins científicos. O CEP concedeu a dispensa do Termo de Consentimento Livre e Esclarecido (TCLE), uma vez que não foram utilizadas entrevistas com pacientes, apenas foram utilizadas cópias das prescrições. O risco da exposição dos dados de pacientes contidos nas receitas foi contro- lado, preservando-se a identificação durante todas as etapas da pesquisa. Também foi concedida a autorização prévia pelo responsável da drogaria para realização da pesquisa.

\section{RESULTADOS E DISCUSSÃO}

Foram analisadas 120 prescrições médicas manuscritas coletadas durante o período de trinta dias na drogaria. No total foram identificados 426 erros. Em relação à legibilidade, as prescrições foram classificadas em legíveis, pouco legíveis ou ilegíveis. Os dados encontram-se pormenorizados na Tabela 1.

Na Tabela 2, podem ser observadas cinco variáveis: identificação do prescritor, identificação do paciente, posologia , dose por unidade posológica e abreviaturas. Os resultados mostram que foram classificadas em completas, incompletas ou omissas. A drogaria, local do presente estudo, realiza o aviamento e retenção de aproximadamente 300 prescrições mensais. A outra parcela é constituída pela venda e dispensação de medicamentos sem retenção de receita ou isentos de prescrição médica. Dessa forma, as 120 prescrições analisadas correspondem a $40 \%$ das 
receitas retidas mensalmente na drogaria. Esse número de prescrições analisadas foi similar ao estudo realizado em uma drogaria localizada no município brasileiro de Montes Claros, MG (1).

Tabela 1. Legibilidade de 120 prescrições atendidas em uma drogaria de São José do Rio Preto, São Paulo, Brasil.

\section{Característica} Classificação Legível

Legibilidade

Pouco legível

Ilegível

Total

Neste estudo, foram identificados 426 erros nas prescrições analisadas. Um estudo transversal realizado na farmácia de um hospital-referência de Minas Gerais detectou um elevado número de erros em prescrições médicas. E considerando o menor nível de complexidade da atenção primária (10)., a taxa de erros nas prescrições analisadas no presente estudo foi considerada alta.

A dificuldade de legibilidade da prescrição tem sido responsável por graves problemas de saúde, sendo inclusive classificada como falta de ética médica. A dispensação de prescrições nestas condições pode levar a erros, com a possibilidade de não ser atingido o efeito terapêutico desejado.

Tabela 2. Características das 120 prescrições atendidas em uma drogaria de São José do Rio Preto, São Paulo, Brasil.

\begin{tabular}{|c|c|c|c|}
\hline Caracteristica & Classificação & $\mathbf{n}$ & $\mathbf{\%}$ \\
\hline & Completa & 108 & 90,00 \\
\hline \multirow{3}{*}{ Identificação do prescritor } & Incompleta & 7 & 5,83 \\
\hline & Omissa & 5 & 4,17 \\
\hline \multirow{3}{*}{ Identificação do paciente } & Completa & 69 & 57,50 \\
\hline & Incompleta & 26 & 21,67 \\
\hline & Omissa & 25 & 20,83 \\
\hline \multirow{2}{*}{ Posologia } & Completa & 72 & 60,00 \\
\hline \multirow{3}{*}{ Dose por unidade posológica } & Incompleta & 20 & 16,67 \\
\hline & Omissa & 28 & 23,33 \\
\hline & Completa & 66 & 55,00 \\
\hline Abreviaturas & Omissa & 20 & 16,67 \\
\hline & Presente & 101 & 84,17 \\
\hline & Ausente & 19 & 15,83 \\
\hline
\end{tabular}

Também pode favorecer o aparecimento de reações não desejadas ou fatais (11).

Nesse estudo, $45,83 \%$ das prescrições foram classificadas como ilegíveis, sendo considerado impossível o entendimento da escrita. Esse número foi superior ao mostrado por Lucas e cols (2012), em Montes Claros, MG;
Azevedo e cols. (2011) em Lavras - MG; e Mastroianni (2009) em Araraquara, SP $(1,11,12)$.

Foram classificadas, nesse estudo, $31,67 \%$ das prescrições como legíveis, isto é, não houve problemas de tempo gasto, além do normal, para entender o que estava escrito; e 22,5\% pouco legíveis, havendo entendimento parcial da prescrição. E esses resultados foram inferiores aos encontrados em Montes Claros, MG (1). Em função da elevada subjetividade e dependência da experiência do avaliador, foi estabelecido um padrão de avaliação o mais homogêneo possível na avaliação da legibilidade, reduzindo aspectos da subjetividade envolvida no julgamento (10).

Quanto à identificação do prescritor, os dados encontrados nesse estudo revelaram que $90 \%$ das prescrições estavam completas, sendo as incompletas 5,83\%, e as omissas, 4,17\%. Pesquisadores em Montes Claros, MG, encontraram resultados semelhantes de prescrições com identificação do prescritor completa, incompleta ou omissa (1). Em relação à variável "identificação do paciente", os resultados foram: completa $57,5 \%$, incompleta $21,67 \%$, e omissa $20,83 \%$, divergente aos resultados do estudo mineiro (1).

Em relação à posologia, $60 \%$ das prescrições apresentaram posologia completa, $16,67 \%$ incompleta e $23,33 \%$ omissa, resultados semelhantes aos encontrados em outros estudos nacionais $(1,10)$. Considerando a dose, os resultados revelaram que $55 \%$ das prescrições apresentaram dosagem completa, $16,67 \%$ incompleta e $28,33 \%$ omissa. Esses resultados também foram considerados congruentes aos estudos supracitados. Em um estudo realizado em Ijuí, RS, a posologia foi ausente em um número menor de prescrições (13). A falta da posologia nas prescrições acarreta administração de doses inferiores ou superiores ao indicado, ineficácia do tratamento e, até mesmo, reações adversas, interações medicamentosas, intoxicações, ou óbito do paciente (14).

Erros relacionados aos medicamentos podem ocorrer em qualquer etapa do processo, ou seja, desde a prescrição até a administração. Neste contexto, uma prescrição médica de qualidade tem papel essencial na prevenção de eventos não desejáveis. Prescrições ambíguas, ilegíveis ou incompletas, falta de padronização, utilização de abreviaturas e presença de rasuras são fatores que aumentam os riscos de erros (15). Nesse estudo, as abreviaturas foram frequentes em $84,17 \%$ das prescrições.

Em relação ao cumprimento da legislação vigente, $65 \%$ das prescrições mostraram-se inadequadas, resultado inferior ao encontrado por pesquisadores em um estudo mineiro (1). Porém, é necessário que todas as prescrições cumpram a legislação de forma integral, sendo este um requisito considerado obrigatório. Os resultados evidenciam o não cumprimento, por grande parte dos prescritores, dos seus deveres e responsabilidades frente o ato da prescrição, acarretando danos à saúde dos pacientes, que ao buscarem soluções para os seus problemas de saúde acabam por desenvolver complicações $(1,10)$. 
A prescrição médica no Brasil é normatizada pela Lei Federal n ${ }^{\circ} 5.991$ de 1973 (17); pela Lei Federal n ${ }^{\circ}$ 9.787 de 1999 (18), e; pela Resolução n 357 de 2001 do Conselho Federal de Farmácia (19). Há também aspectos éticos a serem seguidos descritos no Código de Ética Médica (20) e no Código de Ética da Profissão Farmacêutica (21).

No momento da dispensação de medicamentos, todas as prescrições médicas devem ser avaliadas pelo farmacêutico, observando-se a legibilidade, ausência de rasuras, identificação do paciente, identificação do medicamento, dose, posologia, forma farmacêutica, duração do tratamento, local e data da emissão, assinatura e identificação do prescritor, com o número de registro no respectivo conselho profissional. Além disso, é vetada a dispensação de medicamentos cujas receitas estiverem ilegíveis, e que possam induzir a erro ou confusão. Em caso de dúvidas, o farmacêutico deve consultar o prescritor e solicitar a adequação e substituição da prescrição (16).

Tabela 3. Atendimento à legislação das 120 prescrições atendidas em uma drogaria de São José do Rio Preto, São Paulo, Brasil

\begin{tabular}{|c|c|c|c|}
\hline Característica & Classificação & $\mathbf{n}$ & $\%$ \\
\hline $\begin{array}{c}\text { Cumprimento à } \\
\text { legislação }\end{array}$ & Adequado & 42 & 35 \\
\hline Total & Inadequado & 78 & 65 \\
\hline
\end{tabular}

Considerando que a maioria dos erros envolvendo medicamentos ocorre no estágio de prescrição, a adoção de mecanismos de alerta durante a prescrição apresenta impacto positivo na redução do número de eventos adversos relacionados aos medicamentos e nos gastos em saúde. A falta de observação de todos os critérios das boas práticas na prescrição representa um aspecto negativo no trabalho dos profissionais de saúde, dificultando a conduta farma- cêutica e, oferecendo riscos ao paciente. Para que ocorra uma terapia medicamentosa segura e efetiva, é necessário que médicos e farmacêuticos atuem de forma integrada, utilizando seus conhecimentos para garantir a utilização adequada dos medicamentos $(1,12,13)$.

\section{CONCLUSÃO}

Ao final desse estudo, foi possível verificar elevada taxa de erros em prescrições médicas atendidas na drogaria. Foram identificados erros relacionados a legibilidade, identificação do prescritor e do paciente, posologia e dose, uso excessivo de abreviaturas, além do não-cumprimento da legislação vigente, que preconiza as boas práticas na prescrição.

Estes achados mostram a importância da atuação do farmacêutico na análise das prescrições e na detecção de erros, uma vez que se esses erros passarem despercebidos acarretarão graves consequências ao paciente. Portanto, deve ser destacada a necessidade desse profissional para detecção, solicitação de correções e prevenção dos erros de prescrição, garantindo, assim, a farmacoterapia adequada, a segurança do paciente, além de medidas de farmacoeconomia, contudo, contribuindo para o Uso Racional de Medicamentos.

Cabe, ainda, a melhor divulgação sobre as boas práticas na prescrição e sobre os danos que as prescrições inadequadas acarretam ao paciente. A realização de outros estudos com intuito de criar estratégias para minimizar os erros de medicação relacionados à baixa qualidade das prescrições médicas, bem como, a conscientização da população sobre o direito da atenção à saúde de qualidade, por meio da exigência de prescrições médicas adequadas, devem ser incentivadas, além de uma fiscalização mais atuante nesse âmbito a fim de garantir o cumprimento da legislação.

\section{REFERÊNCIAS}

1. Lucas JCF, Oliveira MC, Fonseca MHG, França DS, Rabelo JA. Avaliação do perfil de receituários médicos coletados em uma drogaria em Montes Claros - MG. Motricidade. 2012; 8(Supl.2):187-196.

2. Maschio-Lima TA, Nakazone MA, Furini AAC. Avaliação Preliminar de Prescrições para Idosos em Serviço de Cardiologia de um Hospital de Ensino. Rev Bras Cardiol. 2014; 27(5):333-341.

3. Cardinal LSM, Matos VTG, Resende GMS, Toffoli-Kadri MC. Caracterização das prescrições medicamentosas em unidade de terapia intensiva adulto. Rev Bras Ter Intensiva. 2012; 24(2):151-156. DOI: 10.1590/S0103$507 X 2012000200009$.

4. Valadão AF, Moreira ALP, Andrade LC, Pires CA, Firmino KF, Brum CA. Prescrição médica: um foco nos erros de prescrição. Rev Bras Farm. 2009; 90(4):340-343.

5. Anacleto TA, Rosa MB, Neiva HM, Martins MAP. Erros de medicação. Pharmacia Brasileira. 2010. [Citado 2015 jul 13]. Disponível em: http://www.cff.org.br/sistemas/geral/ revista/pdf/124/encarte farmaciahospitalar.pdf

6. Lins BG, Cazzamalli F, Zancanaro V. Análises de erros nas prescrições médicas de uma unidade básica de saúde de um município do Meio Oeste Catarinense. RIES. 2012; $1(2): 62-77$.

7. Silva ERB, Bandeira VAC, Oliveira KR. Avaliação das prescrições dispensadas em uma farmácia comunitária no município de São Luiz Gonzaga - RS. Rev Ciênc Farm Básica Apl. 2012; 33(2):255-281.

8. Néri EDR, Gadêlha PGC, Maia SG, Pereira AGS, Almeida PC, Rodrigues CRM, Portela MP, Fonteles MMF. Erros 
de prescrição de medicamentos em um hospital brasileiro. Rev Assoc Med Bras. 2011; 57(3):306-314. DOI: 10.1590/S0104-42302011000300013.

9. Araújo PTB, Uchôa SAC. Avaliação da qualidade da prescrição de medicamentos de um hospital de ensino. Ciênc. Saúde Coletiva. 2011; 16(supl.1):1107-1114. DOI: 10.1590/S1413-81232011000700042.

10. Rosa MB, Perini E, Anacleto TA, Neiva HM, Bogutchi T. Erros na prescrição hospitalar de medicamentos potencialmente perigosos. Rev. Saúde Pública. 2009; 43(3):390398. DOI: 10.1590/S0034-89102009005000028.

11. Azevedo LS, Pereira LJ, Zangerônimo MG, Sousa RV, Murgas LDS, Marques LS, Castelo PM, Pereira CV. Avaliação da adequação legal de receitas e notificações de receita de medicamentos sujeitos a controle especial dos setores públicos e privados. Rev Ciênc Farm Básica Apl. 2011; 32(3):401-417.

12. Mastrianni PC. Análise dos aspectos legais das prescrições de medicamentos. Rev Ciênc Farm Básica Apl. 2009; 30(2):173-176.

13. Frick GGG, Bonotto LF, Bernardi CLB, Pletsch MU. Principais problemas encontrados nas prescrições em município da região noroeste do estado do Rio Grande do sul. Rev Contexto \& Saúde. 2010; 10(19):108-111.

14. Gimenes FRE, Mota MLS, Teixeira TCA, Silva AEBC, Opitz SP, Cassiani SHB. Segurança do paciente na terapêutica medicamentosa e a influência da prescrição médica nos erros de dose. Rev. Latino-Am. Enferm. 2010; 18(6):1055-1061. DOI: 10.1590/S010411692010000600003.

15. Gimenes FRE, Teixeira TCA, Silva AEBC, Opitz SP, Mota MLS, Cassiani SHB. Influência da redação da prescrição médica na administração de medicamentos em horários diferentes do prescrito. Acta Paulist Enferm. 2009; 22(4):380-384. DOI: 10.1590/S010321002009000400005.
16. BRASIL. Ministério da Saúde. Agência Nacional de Vigilância Sanitária. RDC n 44, de 17 de agosto de 2009. Dispõe sobre Boas Práticas Farmacêuticas para o controle sanitário do funcionamento, da dispensação e da comercialização de produtos e da prestação de serviços farmacêuticos em farmácias e drogarias e dá outras providências. D.O.U. Brasília, DF, 17 ago. 2009.

17. BRASIL. Lei no 5.991, de 17 de dezembro de 1973. Dispõe sobre o controle sanitário do comércio de drogas, medicamentos, insumos farmacêuticos e correlatos, e dá outras providências. D.O.U. Brasília, DF, 21 dez. 1973.

18. BRASIL. Ministério da Saúde. Lei n ${ }^{\circ} 9.787$, de 10 de fevereiro de 1999 . Altera a Lei $n^{\circ} 6.360$, de 23 de setembro de 1976, que dispõe sobre a vigilância sanitária e estabelece o medicamento genérico. Dispõe sobre a utilização de nomes genéricos em produtos farmacêuticos e dá outras providências. D.O.U. Brasília, DF, 10 fev. 1999.

19. CFF. Conselho Federal de Farmácia. Resolução nº 357, de 20 de abril de 2001. Dispõe sobre Regulamento Técnico das Boas Práticas de Farmácia. D.O.U. Brasília, DF, 27 abr. 2001 .

20. CFM. Conselho Federal de Medicina. Resolução nº 1.931, de setembro de 2009. Aprova o Código de Ética Médica. D.O.U. Brasília, DF, 13 out. 2009.

21. CFF. Conselho Federal de Farmácia. Resolução no 596, de 21 de fevereiro de 2014. Dispõe sobre o Código de Ética Farmacêutica, o Código de Processo Ético e estabelece as infrações e as regras de aplicação das sanções disciplinares. D.O.U. Brasília, DF, 25 mar. 2014.

22. Sousa LMG, Torres MLD, Melo GC, Magalhães Júnior AA, Firmo WCA. Estudo de prescrições médicas de psicotrópicos de uma farmácia comercial no município de Santa Inês, Maranhão, Brasil. Enciclopédia Bioesfera. 2014; 10(19):2428-2440. 